\title{
IdeAs
}

Idées d'Amériques

$11 \mid 2018$

Modernités dans les Amériques : des avant-gardes à aujourd'hui

\section{Modernité(s) chez Martha Graham et Doris Humphrey, artistes fondatrices de la danse moderne étatsunienne dans les années 1930}

Martha Graham and Doris Humphrey, the Founding Artists of North-American

Modern Dance: an Analysis of the Concept of Modernity

Colocación Norteamérica y modernidad(es) en la danza estadounidense entre

1930 y 1940

\section{Claudie Servian}

\section{(2) OpenEdition}

Journals

Édition électronique

URL : https://journals.openedition.org/ideas/2368

DOI : $10.4000 /$ ideas.2368

ISSN : 1950-5701

Éditeur

Institut des Amériques

\section{Référence électronique}

Claudie Servian, «Modernité(s) chez Martha Graham et Doris Humphrey, artistes fondatrices de la danse moderne étatsunienne dans les années 1930 », IdeAs [En ligne], 11 | 2018, mis en ligne le 02 juillet 2018, consulté le 18 octobre 2022. URL : http://journals.openedition.org/ideas/2368 ; DOI : https://doi.org/10.4000/ideas.2368

Ce document a été généré automatiquement le 18 octobre 2022.

\section{c) (1)}

Creative Commons - Attribution - Pas d'Utilisation Commerciale - Pas de Modification 4.0 International - CC BY-NC-ND 4.0

https://creativecommons.org/licenses/by-nc-nd/4.0/ 


\title{
Modernité(s) chez Martha Graham et Doris Humphrey, artistes fondatrices de la danse moderne étatsunienne dans les années 1930
}

\author{
Martha Graham and Doris Humphrey, the Founding Artists of North-American \\ Modern Dance: an Analysis of the Concept of Modernity \\ Colocación Norteamérica y modernidad(es) en la danza estadounidense entre \\ 1930 y 1940
}

Claudie Servian

1 Cet article se limite à la danse théâtrale étatsunienne dite " moderne » développée dans les années $1930^{1}$ par des chorégraphes installés sur la côte est, Martha Graham(1894-1991) et Doris Humphrey (1895-1958), qui inscrivent leur art dans la modernité pour le distinguer de l'art chorégraphique académique européen et de l'art «denishawnien ${ }^{2}$ » et pour « chercher un art américain de la danse ${ }^{3}$ ».Nous souhaitons réfléchir au concept de modernité à travers les propos de ces deux artistes majeures de l'histoire de la danse étasunienne. Les remarques émises par le critique de danse André Levinson, chroniqueur de la revue L'Art vivant en 1925 ainsi que le recueil constitué d'articles rassemblés par Oliver Sayler, intitulé: Revolt in the Arts: A Survey of the Creation, Distribution and Appreciation of Art in America apportent un témoignage sur les intentions des chorégraphes modernes étatsuniens. André Levinson identifie une renaissance dans la nouvelle expression chorégraphique des années 1930. L'ouvrage d'Henri Meschonnic, intitulé Modernité, constitue une source de réflexion sur cette notion. Notre analyse est également influencée par le travail sur la modernité mené par le Professeur Roland Tissot sur la peinture étatsunienne ${ }^{4}$.

2 Selon Henri Meschonnic, il est difficile de dater les débuts de la modernité en peinture : 
Quant à la peinture moderne, elle naît en France autour des années 1870-1880, selon Bourdieu, qui étudie sociologiquement la 'révolution symbolique' accomplie par Manet et les impressionnistes. D'autres font commencer la peinture moderne à Gauguin. Quand ce n'est pas aux cubistes. En ce sens, c'est entre 1906 et 1908 que commence la modernité en peinture. Pour certains, en 1907, avec les Demoiselles d'Avignon. [...] Mais tel autre, qui étudie le modernisme anglo-saxon en littérature, limite le modernisme à 1910-1930, au 'tournant des années $1930^{5}$ '. Parce que si on ne lui met pas cette borne, si on l'imagine qui continue dans Finnegan's Wake, ou avec l'œuvre de Moore en sculpture, alors, 'le mouvement se perd dans les grandes catégories intemporelles' (ibid.) Cet auteur préfère donc couper l'œuvre de Joyce en deux, après Ulysse, plutôt que de renoncer à circonscrire le mouvement. Précisément, la modernité n'est pas un mouvement, comme dada ou l'imagisme. [...] Pour chaque date, chaque limite, une stratégie, un enjeu. La modernité est le terrain d'un travail du sens dont il n'y a peut-être pas d'équivalent. (Meschonnic, 1988 :25)

3 C'est tout aussi difficile en danse. Les critiques évoquent souvent la date de 1930 comme le début de la danse moderne étatsunienne car elle correspond à l'époque où Martha Graham commence à créer ses propres chorégraphies.

4 À travers la description d'œuvres précises, différentes hypothèses d'interprétation de ce concept seront examinées. On se demandera ainsi s'il relève de l'expérimentation chorégraphique ou, au contraire, d'une stratégie idiosyncrasique ou générique : si les formes prises par la modernité, leurs significations et leurs effets sont propres à un artiste, à un genre de danse ou à une évolution sociale et idéologique des milieux artistiques étatsuniens.

5 Le concept de modernité en danse est difficile à définir tant sa charge idéologique est grande. Meschonnic affirme « ce terme n'a pas de référent fixe, objectif. Il a seulement un sujet. » (Meschonnic, 1988 : 33) Il ne signifie pas la même chose en peinture. En effet, les peintres réalistes, référentialistes et mimétiques attachent une importance considérable au signifié et leur peinture renvoie au traitement de sujets contemporains. En danse, le concept de modernité renvoie à une multitude de projets donnant naissance à une grande variété de formes. Cette analyse s'efforce de porter un éclairage sur cette diversité en étudiant le travail d'artistes choisis pour la réflexion qu'ils ont menée sur la modernité et de faire ressortir le lien entre modernité et art chorégraphique étatsunien.

\section{La recherche des origines}

Pour Henri Meschonnic, «La modernité est un combat sans cesse recommençant parce qu'elle est un état naissant, indéfiniment naissant, du sujet, de son histoire, de son sens. Elle ne cesse de laisser derrière elle les assis de la pensée, ceux dont les idées sont arrêtées, se sont arrêtées, et qui confondent leur ancienne jeunesse avec le vieillissement du monde. La modernité côtoie ce cimetière des concepts fossiles dont nous sommes encombrés. Et qui rendent sourds. Sourds à ce qui vient. [...]» (Meschonnic, 1988: 9) Nous retrouvons dans cette citation des idées formulées par Graham et Humphrey : celle de rejet des codes anciens et celle de conscience du monde dans lequel on vit. Meschonnic poursuit sa réflexion en affirmant que "La modernité est la prévision de ce que c'est qu'être au présent. Le présent, pour la plupart, est tenu par le réseau des intérêts et des pouvoirs, les réseaux des maintiens du passé.» (Meschonnic, 1988 : 13) L'insistance sur le moment présent tout en conservant certains 
acquis du passé constitue un élément de base du travail de ces pères fondateurs de la modernité.

7 L'expression modern dance apparaît aux États-Unis en 1927 grâce au critique John Martin et s'applique alors à des danseurs convaincus que la danse ne doit pas refléter uniquement des comportements ancrés dans la société et ne doit pas être essentiellement une expression de la vie contemporaine. Quant à Susan, elle pense au contraire que «Lorsque le terme 'danse moderne' fut adopté aux alentours de 1927, il convenait parfaitement aux danseurs auquel il était appliqué, car ils pensaient que la danse devrait refléter des attitudes et des préoccupations contemporaines, même lorsqu'ils situaient leurs œuvres à d'autres périodes, comme Graham avec ses pièces traitant de la mythologie grecque. ${ }^{6} »(\mathrm{Au}, 1988$ :119) Les critiques de danse ne sont pas d'accord sur le contenu esthétique ou sociopolitique de la notion de modernité. D'ailleurs, les artistes eux-mêmes conscients des interprétations diverses et variées de leur art lui préfèrent l'expression new dance, nouvelle danse, mieux adaptée à leur volonté de se démarquer de ce qui existe déjà. Martha Graham, l'une des éminentes représentantes de la danse dite "moderne », rejette vivement cette terminologie et lui préfère l'expression "être de son temps ».Elle déclare dans son autobiographie : «La danse moderne se démode très vite. C'est pourquoi j'utilise toujours le terme de 'danse contemporaine', qui est de son temps. Jamais, jamais, je ne parle de 'danse moderne'. Ça n'existe pas. Le public a pu me considérer comme moderne, mais pas moi. ${ }^{7}$ " (Graham, 1992: 198) Meschonnic différencie les termes «moderne» et «contemporain»: «Parce que moderne suppose la subjectivité d'un énonciateur, il ne peut pas se confondre complètement avec la notation temporelle que désigne le contemporain. Moderne ne se borne pas à qualifier une époque. La nôtre." (Meschonnic, 1988 ; 34) John Martin, également conscient que le qualificatif de "moderne » est insuffisant, cherche à déterminer dans son ouvrage The Modern Dances, les caractéristiques de la danse moderne et à en donner une définition, même si elle demeure insatisfaisante :

Le terme de danse moderne est évidemment impropre. Il n'est pas synonyme de danse contemporaine car il ne concerne pas l'ensemble de la création d'aujourd'hui. Il n'est que temporairement exact, si on peut parler d'exactitude, car demain, lorsqu'une forme de danse plus nouvelle sera apparue, il deviendra impossible de se référer à la danse d'aujourd'hui comme moderne. Il reste que ce terme très général a réussi à s'imposer dans tous les arts. [...] Par danse moderne, nous tenterons de suggérer par élimination ces types de danses qui ne sont ni classiques ni romantiques (la danse d'Isadora Duncan et de ses épigones romantiques) [...] La danse folklorique et les danses de spectacles comme les claquettes et la danse populaire acrobatique sont exclues de notre étude ; non qu'elles soient considérées comme inférieures ou sans valeur, mais elles ne font pas partie de la danse en tant qu'art pur. (Martin, 1991 : 20)

8 Comme le souligne John Martin dans cette citation, l'expression "danse moderne " peut renvoyer à une époque ou à un contenu thématique précis ou encore à des points de vue sur le mouvement dansé. Les artistes refusent l'idée d'un style reflet de la modernité car, comme le déclare Jacqueline Robinson, historienne de la danse : «Un style est un phénomène social, une langue, il a sa grammaire, sa syntaxe, c'est-à-dire un système de conventions admises par tous. » (Robinson, $1990: 110)$ Ce n'est pas le cas de la modernité en danse, qui est une mise en question permanente d'elle-même. La danse moderne étasunienne ne possède ni grammaire, ni syntaxe, elle est idiosyncrasique. Cependant, un dénominateur commun permet aux historiens de la danse d'utiliser l'expression "danse moderne" pour évoquer le travail de certains chorégraphes 
fascinés, dès le début du vingtième siècle, par l'idée de renouvellement et de nouveauté. Parmi eux, il y a Martha Graham et Doris Humphrey, toutes deux élèves de la Denishawn', école d'arts californienne fondée par Ruth Saint Denis et Ted Shawn, dont elles critiquaient le côté exotique. Elles ont beaucoup appris auprès de leurs deux maitres, mais pas suffisamment pour satisfaire leur passion. Pour elles, la danse doit provoquer, stimuler, informer mais ne doit pas simplement divertir, elle doit « devenir un art » (Graham, 1992 : 93) à part entière.

9 La modernité chez ces artistes ne relève pas d'une adhésion au modernisme technologique, elle implique au contraire de le combattre en redécouvrant les aptitudes naturelles du corps. Elle renvoie à une recherche des origines. Le fantasme de l'origine se greffe sur ce que l'artiste occidental a perdu au cours de son développement et qu'il faudrait faire resurgir pour enrichir son expérience émotionnelle. Louis Horst, pianiste accompagnateur des cours de danse de la Denishawn à Los Angeles puis compositeur des partitions des chorégraphies de Martha Graham, pense que le danseur moderne sait " retourner dans le passé le plus lointain de l'homme pour aller de l'avant " (Horst, 1961 : 52). Influencée par les interprétations jungiennes ${ }^{10}$ contribuant à propager une vision des fondements primitifs de l'inconscient américain, Martha Graham déclare de son côté " Il arrive un moment où le corps, à travers la danse devient autre chose et accueille tout un monde de cultures passées. Les gestes sont " ancestraux. » (Graham, 1991: 13) Pour elle, chacun est porteur de milliers d'années de mémoire. Nous effectuons des gestes instinctifs, inattendus qui proviennent de souvenirs enfouis. "Très souvent, écrit-elle, la danse vient d'un désir de retrouver ces fresques ensevelies. [...] On ne trouve le passé qu'à partir de soi, à partir de son expérience présente, de ce qui pénètre dans notre existence au moment présent. » (Graham, 1992 : 14) Pour Jung, "le contact avec le primitif ne nous rappelle pas seulement notre enfance, mais notre préhistoire ${ }^{11} »$. En s'intéressant aux primitifs indiens, l'Américain remonterait le fil de ce que son inconscient collectif culturel possèderait de distinctif. Le thème de remémoration de valeurs enfouies et intuitives est une constante dans l'imaginaire des artistes modernes. Persuadés que l'artiste occidental a beaucoup perdu au cours de son développement et qu'il doit remédier à l'appauvrissement de son expérience émotionnelle, les chorégraphes se penchent sur la relation entre mouvement et inconscient. Dans Revolt in the Arts, Graham écrit qu'il faut déterminer «ce qu'est en nous le primitif, cette expression de la psyché de l'Amérique, accessible seulement à un peuple suprêmement cultivé. » (Graham, $1930: 253)$ Toutes les formes de primitif n'ont pas la même valeur de modèle. Pour conquérir une modernité spécifiquement américaine, il s'agit pour les chorégraphes étatsuniens de prendre leurs distances par rapport aux primitifs que les artistes européens ${ }^{12}$ mettent en avant depuis le début du vingtième siècle et donc de s'éloigner des influences des arts africains et océaniens. Dans les années 1930, les artistes étatsuniens insistent sur la nécessité de s'éloigner de l'influence culturelle de l'Europe en cherchant des inspirations primitives autochtones, en s'intéressant aux cultures amérindiennes.

10 La danse moderne permet un retour aux sources, elle donne la possibilité de retrouver l'accès à une vitalité originelle perdue, elle émane de ce qui est caché en l'homme. Elle permet d'exprimer le non-dit, comme chez les Amérindiens qui dansent pour invoquer les esprits de la nature. Les arts tribaux, arts dits "primitifs »et les rituels, envisagés comme une émanation du psychisme primitif, renvoient aux modes d'expression des peuples en dialogue avec la nature qui n'ont pas perdu l'accès au sacré ni au 
symbolique. Le danseur moderne cherche à redécouvrir en lui le primitif, libre de tout conditionnement culturel. L'idée du primitif ${ }^{13}$ rejoint celle d'archaïsme et fait naître chez les chorégraphes citadins modernes un fantasme d'origine. Les références au modèle primitif permettent de reconquérir les aptitudes physiques naturelles de l'être humain, perturbées par l'environnement mécanique. Louis Horst ${ }^{14}$ souligne que « l'art primitif a été de loin l'influence la plus puissante et distinctive pour tous les modernes. » (Horst, 1961: 59) L'idée de "primitif» évoque un état d'enfance de l'humanité qu'il convient de ranimer pour enrichir l'expérience de l'homme moderne. Dès 1906, les Wild West Shows, spectacles de l'Ouest sauvage, font rêver les artistes et le public, par leurs mises en scène des Indiens d'Amérique et de leurs danses. Les arts tribaux sont perçus comme une émanation du psychisme primitif et sont la source d'une grande créativité. John Martin déclare que «l'homme primitif a élaboré une forme de danse qui est le modèle de la danse moderne et est en effet à la genèse de toute forme d'art. » (Martin, 1991 : 59) Dans les arts plastiques également, les fauvistes, les cubistes, les expressionnistes et les dadaïstes, critiques de la culture occidentale, se passionnent pour les arts dits primitifs. Ils rejettent le principe de représentation naturaliste qui domine l'art occidental depuis la Renaissance: ils revalorisent l'art primitif, l'art «nègre» et l'art du Moyen-Âge éloignés des conventions de la représentation mimétique.

11 Grâce à une étude de l'art dit " primitif ", les modernes vont chercher à reconquérir les mouvements naturels contrastant avec l'environnement mécanisé en plein développement. Convaincue que pour créer un art neuf, la danse doit retrouver une dimension rituelle, Martha Graham puise son inspiration dans la source primitive que constituent les danses rituelles des Indiens du Nouveau-Mexique qu'elle découvre lors de voyages. Selon ses propres termes, «Une grande partie de ce que je fais aujourd'hui vient non seulement des Indiens d'Amérique mais aussi des Indiens du Mexique. Non que j'aie tenté de devenir mexicaine ou indienne, mais d'acquérir la capacité de m'identifier à une culture qui n'est pas la mienne.» (Graham, 1992:122) Les danses des Amérindiens sont, à ce titre, intéressantes car elles font preuve d'une créativité spécifique, sacrée et magique qui inspire cette chorégraphes aspirant à redécouvrir un potentiel archaïque et intuitif en s'éloignant des conventions artistiques du vingtième siècle. Graham explique dans son autobiographie : «Les danses des Indiens d'Amérique m'ont toujours fascinée, de même que ces instants magiques juste avant le lever du soleil dans les pueblos, ou ma première vision des femmes hopi avec leurs coiffures de fleurs écrasées dont je devais me servir pour Appalachian Spring." (Graham, 1992: 149)Avec Erick Hawkins, compagnon et danseur de la compagnie, elle fréquente des tribus indigènes d'Amérique mais elle ne danse jamais de danse ethnique. Elle trouve auprès des Indiens une forme d'éveil " une grâce, un émerveillement, mais jamais je n'ai dansé une de leurs danses, écrit-elle.» (Graham, 1992: 150) En 1931, dans sa chorégraphie Primitive Mysteries, créée après un séjour au Nouveau-Mexique où elle assiste à des danses cérémonielles, Graham utilise un mélange de religion chrétienne et de culture indienne. Elle propose la mise en scène d'un rituel en l'honneur de la Vierge Marie comportant trois épisodes : la naissance de Jésus, sa crucifixion, et l'Assomption. La soliste, vêtue de blanc, utilise les forces gravitationnelles des danses amérindiennes : elle chute au sol, elle s'assied ou s'allonge sur le plateau, elle se tient à genou, comme pour prier, elle effectue des mouvements simples, asymétriques et saccadés. Dans les danses rituelles, les distorsions reflètent l'angoisse de l'homme face aux éléments. L'inspiration amérindienne se traduit dans la qualité terrienne et cérémonielle de ces 
danses. Les danseurs martèlent le sol, s'y enfoncent car « Le sol est sacré partout, dans cette région. Sacré pour les Indiens, sacré même avant qu'ils s'y installent. Quels animaux étranges vivaient ici, quelles plantes étranges. Tout cela repose sur un sol sacré. Un sol consacré à la vie, aux merveilles de la vie.» (Graham, 1992: 226) La danseuse soliste est entourée par douze autres interprètes renvoyant aux effets de masse des danses rituelles amérindiennes. La chorégraphe observe ces danses autochtones avec une grande concentration car elle cherche à comprendre les moyens expressifs qui permettent aux Amérindiens de transcrire leurs croyances. Elle repère une structure répétitive qu'elle reprend, donnant à la danse grahamienne un effet incantatoire, elle remarque le pouvoir expressif du mouvement rituel détaché de toute signification mimétique. La danse amérindienne rompt avec les représentations réalistes du monde pour utiliser les effets impersonnels de l'expression symbolique afin d'aboutir à une abstraction formelle. Stylisée, la danse s'empare de l'intensité spirituelle des rituels. L'importance des marches lourdes évoque la dévotion des Indiens pour leur terre. En s'immergeant dans cette culture, Graham renoue avec ce qu'elle pense être une danse américaine. En éveillant cette mémoire primitive, Graham retrouve les racines de l'inconscient américain. L'influence des Indiens se manifeste aussi sous forme narrative dans American Document (1938) dans laquelle intervient le personnage d'une jeune Indienne. Dans Appalachian Spring (1944), la jeune Indienne " est une figure symbolique du pays, l'Ève de notre Genèse. ${ }^{15}$ "

Les représentations du primitif marquent, dans les années 1930, les préoccupations des chorégraphes modernes. Au cours des premières décennies du vingtième siècle, les termes de "primitif», « rituel», " origine» sont des mots clés du vocabulaire des danseuses modernes, Graham et Humphrey, révélateurs de la volonté de ces chorégraphes d'ancrer leur expression dans les dimensions psychiques, archaïques et universelles de l'existence humaine afin de trouver des formes artistiques inédites. Merle Armitage cite la remarque de Martha Graham à propos de la notion de "primitif »: «car en tant que nation, nous aussi nous sommes primitifs, primitifs au sens où nous formons une nouvelle culture. » (Armitage, 1978 : 100) Cette notion, chez Graham, est paradoxalement liée à celle de l'américanité contemporaine. La façon de traiter les émotions individuelles et collectives par des conduites rituelles décline dans le monde contemporain. Réveiller le primitif signifie retrouver les sources de l'expérience du mouvement. Humphrey partage les mêmes convictions et déclare que "Nous disposons d'un formidable héritage gestuel qui remonte à l'homme primitif. ${ }^{16} »$ (Lloyd, 1949 : 86) Depuis toujours, le mouvement exprime une réaction psychique à l'environnement. C'est cette corrélation entre mouvement et affect, au cœur des danses rituelles, que le danseur moderne doit utiliser pour produire de nouvelles formes artistiques. Humphrey et Graham cherchent à redécouvrir les facteurs fondamentaux de l'expérience du mouvement en ayant, par exemple, recours à un rythme répétitif constituant un outil privilégié pour aborder un état de transe hypnotique. La répétition et le contraste permettent de mettre en valeur tel ou tel aspect du mouvement. Grâce aux modifications subtiles du corps, le danseur devient alors capable de s'exprimer. D'autre part, pour communiquer ses émotions, le danseur moderne peut utiliser la symbolisation qui relève d'un processus d'abstraction comparé à celui que les peintres modernes européens admirent dans les sculptures et les masques africains. 


\section{Le mythe de l'avant-garde}

Pour ces chorégraphes dits "modernes", la danse est une forme artistique inscrite dans l'histoire de l'humanité, elle procède d'une généalogie, mais elle est aussi la construction de ce qui est à venir. Les artistes étatsuniens se perdent dans le mythe du renouveau à tout prix. Leur envie d'innover devient un véritable syndrome ${ }^{17}$. Pour les chorégraphes dits "modernes", il ne faut pas regarder en arrière. Martha Graham déclare «c'est ça la vie pour moi. La vie ne consiste pas à abandonner mais à aller de l'avant. Qu'ai-je d'autre à faire que d'aller de l'avant ? C'est ça la vie pour moi. Ma vie. » (Graham, 1992 : 145) Helen Tamiris, citée par Jack Anderson pense que « tous possèdent une énergie qui les incite à pousser de l'avant. " (Anderson, 1992: 54) Les modernes explorent de nouveaux territoires car un art n'est vivant que s'il évolue. Graham déclare à ce propos: "Mais le changement est la seule constante. [...] Je crois au changement parce qu'il est éternel. Il ne reste jamais en place, il est croissance éternelle, éternelle renaissance.»(Graham, 1992: 89) Pour elle, le présent et le changement sont liés : «Ma seule passion, c'est le travail, c'est ce que Saint-John Perse appelle naître à l'instant, à maintenant. C'est devenir une partie de cette constante dans la vie, notre unique constante: le changement. (Graham, 1992: 216) Elle ne pense pas qu'il y ait vraiment de précédent en art car chaque instant est nouveau, «débordant d'espoir». (Graham, 1992: 216) Un art tourné vers le passé serait condamné à une existence éphémère et deviendrait la négation de l'art. L'artiste étatsunien est pris dans cette obsession du renouvellement continu et devient le représentant de l'original à tout prix. Toute présomption d'influence est suspecte : les chorégraphes modernes récusent l'image de l'arbre généalogique dynastique. Ils questionnent les systèmes existants pour les pervertir en mettant en avant le désir d'originalité de l'artiste moderne. Ce désir s'articule à toute une série de comportements fondés sur la rupture. La critique Marcelle Michel cite les mots de Louis Horst qui souligne à juste titre le fait que « l'Américain est possédé du désir de bouger, de partir, de découvrir de nouveaux horizons. Nous sommes sans cesse à la recherche d'un espace vital. Notre regard est porté vers le lointain.» (Michel, 1984:21) Cette remarque convient au chorégraphe moderne étatsunien soucieux de transformer son art et d'aller de l'avant.

14 La modernité du danseur étatsunien s'exprime dans son souhait de s'émanciper de la sujétion intellectuelle européenne, de rompre avec la tradition. Comme le dit Aaron Osborne, cité par Daniel Dobbels, «Les Américains sont révolutionnaires dès le départ. Ils sont Américains parce qu'ils se sont détachés de l'Europe et qu'ils ont rompu ou ont été obligés de rompre avec les traditions.»(Dobbels, 1995: 54) Les chorégraphes étatsuniens rejettent les conceptions européennes jugées passéistes et inadaptées à la vie américaine. Ils souhaitent se détourner des conventions artistiques du siècle précédent et prendre un nouveau départ. Inspirés par les idées émises par quelques écrivains du XIXe siècle, ils pensent que l'Amérique ne doit pas être une représentation du Vieux Continent. Ralph Waldo Emerson (1803-1882), lors d'un discours en 1837, soutient l'indépendance littéraire des États-Unis et recommande aux Américains de créer leur propre style, libéré des influences européennes. L'auteur développe l'idée d'une spécificité américaine allant de pair avec le refus des contraintes et des canons artistiques : un artiste doit créer selon son inspiration en choisissant les formes qui lui conviennent. Comme les écrivains, les chorégraphes étatsuniens sont désireux de créer 
une danse débarrassée de l'académisme, une danse différente, expressive et multiple. La danse moderne, «le phœnix de l'art " (Anderson, 1992: 165), selon l'expression adoptée par Helen Tamiris, citée par Jack Anderson, comme le phœnix mythique, renaît toujours de ses cendres en rompant sans compromis avec le passé. Elle se transforme tout le temps, elle possède une extraordinaire faculté de renouvellement.

\section{Le corps moderne : le miroir de la pensée}

La modernité en danse est à chercher aussi dans le mouvement. Pour le danseur moderne, tout mouvement ou kinésis, est intrinsèquement lié à une intention mentale ou métakinèse. Le danseur exprime des expériences mentales et émotionnelles par le mouvement corporel. La connexion permet au mouvement de produire de l'émotion chez celui qui danse et celui qui regarde. C'est dans le territoire de l'inconscient et des pratiques rituelles que le critique John Martin $^{18}$ situe le champ d'exploration de la danse moderne. Du point de vue formel, les chorégraphes modernes sont préoccupés par leur désir d'inventer de nouvelles structures et de nouvelles techniques permettant d'exprimer leur âme à l'heure de la vogue de la psychanalyse. C'est sans doute dans cette célébration du mouvement, de sa dynamique propre, de son rythme interne que les chorégraphes étatsuniens ont jeté les bases de la danse moderne.

Le geste est au centre de la théâtralité moderne et de la rhétorique des corps, il peut révéler un état émotionnel ou psychique. Les gestes de la vie sont transposés en mouvements dansés parce qu'ils sont animés de l'intérieur par la force de la civilisation et d'un monde en train de se développer et de se moderniser. L'idée de redonner à la danse son pouvoir d'expression et de communication rejoint les grands courants de l'art moderne, comme celui de l'expressionnisme en Allemagne, pour lequel la mission de l'art est de faire pénétrer les hommes au cœur de l'être et de vibrer. Déjà au début du vingtième siècle Isadora Duncan déclarait que « Le corps doit devenir translucide, et n'est que l'interprète de l'âme et de l'esprit. ${ }^{19}$ " Les chorégraphes Graham et Humphrey expriment leur expérience émotionnelle par les mouvements. Leur danse est le résultat d'un mouvement intérieur, provenant des émotions, des sensations et de l'imagination. Martha Graham réalise le rêve de Noverre ${ }^{20}$, « inventer un langage du corps capable de parler de l'âme, permettre au ballet d'égaler la tragédie.» L'émotion devient une espèce de stimulus donnant au mouvement sa raison d'être. Dans un même esprit, Doris Humphrey déclare que « l'art moderne est l'annexion de formes soumises à un schéma intérieur." (Humphrey, 1990: 43) Tout un courant influencé par le metteur en scène Constantin Stanislavski ${ }^{21}$ et son théâtre de comportement se développe au moment où Graham et Humphrey réfléchissent sur leurs objectifs esthétiques. Convaincu de l'importance de l'expressivité corporelle pour le jeu des acteurs, Stanislavski s'interroge sur les enjeux d'une chorégraphie théâtrale, et met au point une méthode permettant aux interprètes d'explorer les profondeurs de l'inconscient et de vivre de l'intérieur les sentiments des personnages qu'ils représentent. Une aubaine pour les artistes étatsuniens en quête d'identité. La danse devient le théâtre de l'âme.

La modernité du corps moderne est inscrite dans une conception essentiellement expressionniste. Le corps est assigné à exprimer une intériorité, comme chez Martha Graham et à représenter ou illustrer un fait social comme chez Doris Humphrey. Dans tous les cas, le corps est traité de façon symbolique et devient un matériau expressif et signifiant. Le mouvement stylisé et construit exprime un état émotionnel sans réalisme 
mais avec éloquence. La forme dans l'espace, plus que tout autre facteur du mouvement, donne à voir l'intention, le climat et fait percevoir le sens. Le corps moderne devient le miroir de la pensée, le mouvement constitue la première réaction normale à toute expérience mentale et émotionnelle. La danse grahamienne donne une forme objective à la subjectivité de la vie intérieure, mais la forme pour la forme ne satisfait pas le sens esthétique de l'artiste. Dans Deep Song, solo créé en 1937, Martha Graham, vêtue d'un costume en jersey qui souligne ses mouvements amples, souhaite traduire une intensité dramatique extrême. Alice Helpern cite les mots de la chorégraphe : «Je voulais le mouvement significatif. Je ne voulais pas qu'il soit beau ou fluide, je voulais qu'il soit chargé d'une signification intérieure et d'une vive émotion. » (Helpern, 1994: 9) Graham donne aux mouvements un sens symbolique. La technique expressionniste de cette artiste est introspective. Graham répète que le mouvement ne ment pas et que la danse peut aller plus loin que les mots : " Chacun de nous raconte sa propre histoire même sans parler. Le mouvement ne ment pas. » (Graham, 1992 : 12) Les mouvements de Deep Song sont adaptés pour exprimer la douleur des femmes endeuillées lors de la guerre d'Espagne. Les interprètes se tordent à l'oblique, comme l'explique l'historienne de la danse Ernestine Stodelle,

La danseuse est arquée, bien au-dessus du banc, le visage enfoui dans la paume de main. Puis, tel un animal blessé, elle s'est projetée vers l'avant pour se tordre et ramper en une frénésie de désespoir. Suivirent une série de chutes, certaines se brisant vers l'arrière, certaines à demi suspendues comme si paralysées dans un geste de torture. (Stodelle, $1962: 70$ )

Les mouvements au sol tels les rampements, les torsions sont interprétés comme expression de la douleur physique et morale, ils sont chargés d'une tension dramatique. Martha Graham demande aux danseurs d'entreprendre une introspection à une époque où l'Amérique vit à l'heure de la psychanalyse.

Comme Graham, le danseur moderne, en cherchant à se dépasser, se situe entre l'extase et la douleur, il extériorise son âme par le mouvement. Le corps du danseur représente un au-delà du corps, un corps métamorphosé. En dansant Ekstasis (1933), signifiant " action d'être hors de soi » en grec, Graham libère ses élans intérieurs par la danse. Cette idée de dépassement de soi, développée par Nietzsche dans La Naissance de la tragédie (1872), où Nietzsche/Zarathoustra affirme que l'idée même de l'au-delà est née du corps, a été reprise, au début du vingtième siècle par Isadora Duncan, qui parle de «l'extase dionysiaque » exprimée par la danse. Nietzsche perçoit que l'idéal grec de modération et d'équilibre en toute chose est apollinien, mais les rites dionysiaques offrent une issue à la monotonie. Cette tension entre deux pôles complémentaires, mise en avant par le philosophe, se retrouve tout au long de l'histoire de la danse. L'auteur attache une importance considérable à l'opposition de l'apollinien et du dionysiaque dans les grands mythes fondateurs. Cette idée est reprise par Sondra Horton Fraleigh qui distingue la forme et l'expressivité en déclarant que « la forme et l'expressivité sont aussi mises en contraste dans les figures des dieux grecs Apollon et Dionysos. Le dieu Apollon règne sur la forme ; à travers lui, les modèles se forgent. Á l'opposé d'Apollon, Dionysos, dieu du chant et de la danse.» (Horton Fraleigh, 1987 : 115) Ces oppositions sont reliées dialectiquement. La danse américaine, par son dynamisme, renvoie à l'excès, à la démesure, au dionysiaque, elle semble revenue à son rôle premier de transe sacrée. La chorégraphie Celebration (1934) de Graham est une explosion joyeuse de mouvements rapides et énergiques, de sauts nerveux et élastiques. Ce caractère d'extériorisation se rapproche des danses des hommes primitifs qui dansaient pour 
exprimer des sensations au-delà de la raison. Toute transe se constitue à travers cette bipolarité apollinienne et dionysiaque. La danse classique appartient à la tendance apollinienne, au rêve, à la juste mesure, à l'apparence, au masque ; la danse moderne s'intègre à la conception dionysiaque en mettant en valeur l'ivresse, la joie du mouvement permettant d'exprimer l'égarement, l'état extatique dont parle Isadora Duncan. Martha Graham pense également que la danse est le théâtre de l'extase. Ellemême est sauvage quand elle danse, elle est comme " une fleur sauvage dans un jardin de jazz » (Graham, 1991: 96). Elle est animale dans ses mouvements : elle explique qu'elle voulait « être une créature sauvage et belle, d'un autre monde peut-être mais très sauvage. » (Graham, 1992 : 72)

Pour un chorégraphe moderne, la danse ne doit pas être mimétique, comme pour la pantomime. La danse doit évoquer, suggérer avec des formes adaptées à l'expression d'une impulsion intérieure. Le mouvement devient l'indicateur de l'âme. Le corps est libéré des contraintes de l'académisme mais d'un autre côté, il est soumis à des lois tout aussi rigoureuses de respiration, de contraction et de détente, chez Graham; de chute et de récupération, chez Humphrey. Peut-on raisonnablement évoquer un académisme de la modernité dans la danse étatsunienne des années 1930 ? Un académisme exigerait une technique commune, des codes communs, or la danse moderne est le reflet de la personnalité de chaque artiste, ce n'est pas une technique de danse, c'est un point de vue sur l'art. On ne peut pas parler de technique moderne comme on parle de technique classique. La danse de ces créateurs correspond à un état d'esprit sans aboutir à une codification des mouvements. Il n'y a pas de style moderne, mais il existe une esthétique moderne basée sur l'asymétrie, la dualité.

Pour exprimer la démesure à la fois géographique, à l'image de l'Amérique, psychologique et comportementale, les danseurs font des mouvements asymétriques car les mouvements symétriques expriment l'ordre et la raison, or il faut exprimer des passions. Les formes symétriques répondent à un besoin d'équilibre, « une forme en soi possède toujours un sens, écrit Doris Humphrey, le dessin symétrique suggère toujours la stabilité, le repos, un état sans passion. » (Humphrey, 1990 : 181) L'asymétrie stimule les sens. Les formes symétriques et asymétriques se divisent en opposition ou en succession, leurs lignes s'opposent entre angle droit, suggérant la vitalité et la force, et courbes fluides. Pour cette artiste, les contrastes font partie de sa philosophie du mouvement: équilibre/déstructuration, agitation/repos, danger/paix. L'homme est partagé entre ces deux tendances : l'envie de la perfection et la stabilité et à l'opposé un désir d'abandon. Elle nomme sa théorie «l'arc-entre-deux-morts » : l'opposition entre l'horizontal et le vertical, entre tomber et se ressaisir, entre équilibre et déséquilibre. Sa danse est à la fois libérée, libérée de la musique, du spectaculaire, et assujettie aux exigences d'une structure formelle cohérente, et à l'expressivité. Quoique sa technique fût basée sur ces principes-clés, elle ne fut jamais codifiée comme le fut celle de Martha Graham. Chez Humphrey, il y a une recherche constante du mouvement neuf, dicté par le sujet de l'œuvre. Dans Water Study (1928), elle utilise la chute et la reprise pour suggérer le mouvement des vagues. Dans Drama of Motion (1930), elle abandonne la musique et se concentre sur les éléments formels: le rythme et la dynamique qui donnent naissance au thème de la chorégraphie.

22 Alors que les chorégraphes classiques demandent aux danseurs de dissimuler le souffle et l'essoufflement, le danseur moderne réintègre le pouvoir expressif du souffle et se sert de la respiration pour créer de nouveaux mouvements car la respiration est le 
mouvement premier du corps. L'acte de respirer représente le point de départ de l'acte de danser. Le flux du mouvement reflète le flux de la respiration. Martha Graham explique qu'elle voit «ce que fait le corps lorsqu'il respire. Quand il inspire, c'est un release et quand il expire, une contraction. On inspire et on expire. C'est l'usage physique du corps en action.»(Graham, 1992: 46) Chez Graham, il y a un mouvement de contraction en soi suivi d'une expansion vers le monde. La contraction représente un mouvement vers quelque chose, ce n'est pas une position. "C'est un mouvement, explique-t-elle, intrinsèquement dramatique, un moyen pour exprimer le paysage intérieur.» (Graham, 1992 : 23)

Pour les modernes, le jeu avec la force de la gravité et l'interaction avec le sol sont inhérents à l'expression. Alors que la danse classique utilise l'élévation, la danse moderne utilise le sol. Martha Graham respecte le sol tout comme les Amérindiens respectent la terre. Plus le danseur cherche à entrer en contact avec la terre mère, plus son regard est tourné vers l'intérieur, plus son voyage est introspectif et lui donne la possibilité de se découvrir lui-même et de découvrir les autres par projection. Pour Doris Humphrey, «toute la vie fluctue entre la résistance et l'abandon à la gravité. » (Humphrey, $1990: 122)$ Sa technique du fall and recovery introduit des notions de poids, de rebond et de suspension. Elle utilise le corps comme un rebondissement perpétuel entre la position verticale et le contact avec le sol. La notion de conflit est inhérente dans le drame musculaire qui se joue entre l'équilibre et le déséquilibre. La chute ne laisse pas le corps à terre, elle contient en elle le rebond, comme la verticalité recèle l'essence de ce qui va trébucher. Dans son solo Two Ecstatic Themes (1931), elle fait alterner de langoureuses spirales et des poussées agressives vers le haut évoquant la lutte pour rester en équilibre afin de tenter de conserver la vie menacée par des forces extérieures hostiles. Le point d'appui de tous les mouvements se situe dans la région pelvienne et génitale, car il y a la conjonction entre deux grandes lignes de force de vie: vie de l'individu dans la respiration, vie de l'espèce dans la sexualité. Tout mouvement procède $d u$ centre $d u$ corps vers la périphérie, unifiant l'être et l'empêchant de se disperser.

\section{Une danse référentielle}

Meschonnic déclare que «la modernité est critique, elle est provocation» (Meschonnic, 1988:17) La modernité s'exprime aussi à travers une danse moderne référentielle, comme la peinture qui utilise l'histoire contemporaine comme source d'inspiration. L'évolution de la danse étatsunienne des années 1930 enregistre les effets d'un contexte. Alors que certains chorégraphes explorent une expression abstraite à valeur universaliste, d'autres élaborent des danses narratives en prise directe avec l'actualité contemporaine. Ces danseurs utilisent leur environnement social et idéologique pour développer de nouvelles conceptions artistiques. La terre étatsunienne, terre de paradoxes, exerce une influence considérable sur les artistes préoccupés à la fois par la tradition et la modernité. Pour Doris Humphrey, « la danse moderne est née au et du vingtième siècle, elle cherche sincèrement à dire notre temps, notre manière de penser, nos rêves. " (Humphrey, 1990 : 194) Les chorégraphies nées alors traduisent des réactions face aux transformations de l'environnement. Elles repensent le monde contemporain dans ce qu'il a de déstructurant. 
La danse étatsunienne moderne retrace l'histoire américaine, elle nous conte une histoire américaine. L'histoire de Frontier (1935), chorégraphiée par Martha Graham, commence avec Christophe Colomb et se termine au moment de la crise de 1929. La danse est accompagnée de textes écrits, fragments de textes historiques. Dans The Puritan Episode, Martha Graham utilise The Song of Myself de la Bible ainsi que des sermons. Dans d'autres passages, elle sélectionne des extraits de La Déclaration d'Indépendance, des poèmes de Walt Whitman, des discours d'Abraham Lincoln et de $\mathrm{F}$. D. Roosevelt pour enraciner sa danse dans un contexte historique précis, pour renvoyer à des épisodes connus de l'histoire étatsunienne et ainsi ancrer son récit et son œuvre dans le référentiel. Les décors d'Isamu Noguchi sont inspirés par la fascination exercée sur Martha Graham par les nouveaux territoires, symboles du voyage vers l'inconnu. Elle explique que "dans le train vers la Californie, les rails interminables avaient évoqué pour moi cette frontière.» (Graham, 1992: 183) Le décor est sobre, un banc symbolise une barrière, deux cordes simulent les rails qui fuient au loin. Cette chorégraphie est un clin d'œil aux Américains, à leurs ancêtres colons, pionniers et à leurs origines.

Les chorégraphes traitent également de sujets contemporains. Créateurs attachés au contexte extérieur, ils prennent la société américaine, l'histoire américaine ou l'histoire contemporaine comme référents. Créateurs engagés: beaucoup militent, pendant la période de la crise de 1929, avec pour objectif le désir d'une réalisation effective des promesses passées des Pères Fondateurs, pendant que d'autres prennent leurs distances par rapport à l'engagement militant et se réfèrent aux archétypes structurant les récits mythiques de l'humanité pour parler un langage universel. Martha Graham appartient aux deux catégories et s'intéresse particulièrement aux problèmes contemporains qui secouent l'Amérique. Le critique Jack Anderson explique que :

Pendant la Grande Dépression, de nombreux chorégraphes ont créé des danses de contestation sociale. Martha Graham était rarement ouvertement politique mais pendant les années trente et les années quarante, elle a effectivement chorégraphié des œuvres concernant certaines des forces qui ont aidé à façonner la culture américaine ${ }^{22}$. (Anderson, $1992: 176$ )

En 1927, l'année des grandes manifestations de l'Union Square contre l'exécution de Sacco et Vanzetti, elle crée Revolt, un solo sur une musique d'Arthur Honegger. La danse et la musique contribuent à peindre une âme à l'agonie, déprimée, opprimée, cherchant en vain à se libérer de toute forme de tutelle, de contrainte, de coercition. En pleine période de récession, elle monte Steps in the Street, pièce inspirée par le chômage et la crise en 1936, témoignage du monde des exclus de la société. Elle mélange la simplicité de la présentation et la complexité de la structure. Sa forme est abstraite. Elle dénonce les injustices, les oppressions. Elle refuse de participer aux Jeux olympiques de Berlin en tant que chorégraphe invitée :

Il me parait impossible de danser en Allemagne actuellement. Tant d'artistes que je respecte et que j'admire ont été persécutés, privés du droit de travailler pour des raisons ridicules et insatisfaisantes, que je considérerais comme inadmissible de m'identifier, en acceptant cette invitation, au régime qui a permis de telles choses. (Graham, 1992 : 131)

Doris Humphrey, citée par Paul Bourcier, suit le même chemin en réclamant une danse enracinée non dans un passé imaginaire mais dans la nation américaine : 
La danse d'action nouvelle doit naître du peuple qui a su dominer un continent, ouvrir des myriades de chemins à travers les forêts et plaines, vaincre les montagnes, bâtir des tours d'acier et de verre. La danse américaine est le résultat de ce monde nouveau, de cette vie et cette vigueur nouvelles. (Bourcier, 1994 :73) d'une époque. Pour Humphrey et Graham, ce n'est pas en juxtaposant de manière éclectique des emprunts faits à d'autres formes que l'on peut construire une œuvre américaine. Elles critiquent toutes deux l'exotisme de la Denishawn et s'en éloignent. Elles estiment que le chorégraphe moderne doit être de son temps et de son pays. Humphrey pense que la Première Guerre mondiale a joué un rôle déterminant dans l'évolution artistique occidentale. Les bouleversements sociaux provoqués par ce cataclysme mondial furent un facteur déterminant dans l'avènement des théories de la composition :

Tout fut réévalué à la lumière de la violence et de la terrible désintégration, et la danse n'y échappa pas. Deux foyers du monde réagirent avec une force particulière: aux États-Unis et en Allemagne, les danseurs se posèrent des questions sérieuses : «Que raconte ma danse ? Est-ce que cela correspond à ce que je suis et au monde dans lequel je vis? » (Humphrey, $1990: 26$ )

Dans The Life of the Bee (1929), la danseuse dramatise l'étude de Maurice Maeterlinck (1862-1949) sur l'autorité hiérarchique de la reine des abeilles qu'il compare aux devoirs des abeilles travailleuses. Dans cette chorégraphie symbolique, Humphrey critique la structure sociale hiérarchique. La chorégraphe observe et transpose la vie américaine grâce au mouvement dansé. Les évolutions de la danse enregistrent les effets d'un contexte. Les facteurs extérieurs viennent affecter la forme et le contenu de la danse à un moment historique donné.

\section{Conclusion}

31 La modernité dans la danse américaine dans les années 1930 ne s'enferme pas dans le singulier, elle est multiple. Certains chorégraphes se mobilisent pour créer des danses expressives, d'autres pour élaborer des formes narratives en prise avec l'actualité contemporaine, d'autres encore développent une danse abstraite à valeur universelle. Cette notion renvoie à une attitude par rapport à la danse, un état d'esprit, elle encourage l'individualisme artistique et développe des styles chorégraphiques particuliers et idiosyncrasiques. Il n'existe aucune règle établie, chaque artiste crée ses propres codes, de même qu'il n'existe pas à proprement parler de courant moderne pour la danse, pas d'académisme moderne, car chaque artiste a ses propres valeurs, fait ses propres choix en fonction de son vécu et crée une danse personnelle. Les modernités de la danse étatsunienne se déclinent de plusieurs façons donnant à la notion même de modernité un aspect protéiforme très complexe. Graham s'intéresse à l'individu, Humphrey aux interactions entre l'individu et le groupe.

Tous les modernes, cependant, considèrent le ballet académique comme un genre faux et décadent. Ils rejettent les codes pour partir à la conquête des principes fondamentaux et fondateurs des mouvements. Les chorégraphes étatsuniens sont des pionniers de l'innovation mais ne conçoivent pas un courant spécifique doté d'un style spécifique et d'une technique spécifique. Ils ont en commun une certaine conception de l'art, expression de la personne et de son individualité. Ces artistes, farouches défenseurs de la notion protéiforme de modernité en danse, unissent des 
inconciliables : le passé et l'avenir, l'abstraction et l'expression, le subjectif et l'objectif. Cette volonté de concilier les contraires, de résoudre les dichotomies, qui déchirent une nation, imposées par le rationalisme et la logique du peuple américain est une constante de l'esthétique de ces artistes. Leur danse est ambivalente, comme le tourbillon de Mallarmé, symbole de conciliation des contraires. Pour le poète, cité par Jean-Paul Richard, le tourbillon est un moment de "synthèse absolu », il satisfait à la fois « à un vœu de synthèse et à une ivresse d'envol. » (Richard, 1961 : 430) Sous le signe de la danse tournoyante, le songe bouleverse la réalité, le merveilleux accepte la science, l'Antiquité le Nouveau Monde. La modernité est une mise en question permanente de soi-même, elle est l'expression d'un self-made choreographer.

\section{BIBLIOGRAPHIE}

Anderson, Jack, Ballet and Modern Dance, A Concise History, Princeton, New Jersey, A Dance Horizons Book, 1977, 2éd., 1992.

Armitage, Merle, Martha Graham: The Early Years, New York, Da Capo Press, 1978.

$\mathrm{Au}$, Susan, Ballet and Modern Dance, London, Thames and Hudson Ltd, 1988.

Bourcier, Paul, Histoire de la danse en Occident, Paris, Seuil, 1994.

Cunningham, Merce, « Entretiens avec Jacqueline Lesschaeve », Le Danseur et la danse, Paris, Pierre Belfond, 1980, p. 170-171.

Dobbels, Daniel, « L'Héritage Humphrey/Limòn”, Nouvelles de danse, Bruxelles, Contredanse, 1995, p. 54 .

Duncan, Isadora, « La danse et la nature », Écrits sur la danse, Paris, Éditions du Grenier, 1927, p. 24.

Dussurget, Pascaline, «L'enfant Graham », Danser, Paris, SPER, n 137, 2002, p. 31.

Horton Fraleigh, Sondra, Dance and the Lived Body. A Descriptive Aesthetics, Pittsburgh, Pittsburgh University Press, 1987.

Graham, Martha, Blood Memory, New York, Doubleday, 1991.

Graham, Martha, Mémoire de la danse, Arles, Actes Sud, 1992.

Helpern, Alice, The Technique of Martha Graham, New York, Morgan and Morgan Press, Dobbs and Ferry, 1994.

Horst, Louis, Carroll Russell, Modern Dance Forms in Relation to the Other Modern Arts, Princeton, New Jersey, Princeton Book Company, 1961, p. 52.

Humphrey, Doris, The Art of Making Dances, Alton, Dance Books Ltd, 1959.

Humphrey, Doris, Construire la danse, traduit par Jacqueline Robinson, Paris, Bernard Coutaz, 1990. 
Humphrey, Doris, «What a Dancer Thinks About”, The Vision of Modern Dance in the Words of its Creators, Highstown, Princeton Book Company, 1998, p. 59.

Lloyd, Margaret, The Borzoi Book of Modern Dance, New York, Dance Horizons, 1949.

Martin, John, La Danse moderne, traduction Sonia Schoonejans et Jacqueline Robinson, Arles, Actes Sud, 1991.

Martin, John, The Modern Dance, New York, A.S.Barnes and Co., Inc., 1933.

Meschonnic, Henri, Modernité, Paris, Gallimard, Folio essais, 1988.

Michel, Marcelle, « L'Élaboration d'une tradition », Programme de la première biennale de la danse Lyon, Lyon, Rapid Copy, 1984.

Richard, Jean-Paul, L'Univers imaginaire de Mallarmé, Paris, Seuil, 1961.

Sayler, Oliver, Revolt in the Arts: A Survey of the Creation, Distribution and Appreciation of Art in America, New York, Brentano's, 1930.

Siegel, Marcia B.,» L’Autre mère », Nouvelles de Danse, été 1995, p. 75.

Soupault, Philippe, Terpsichore, Paris, Hazan, 1928, 1986.

Stodelle, Ernestine, "Midstream, The Second Decade of Modern Dance: Martha Graham", Dance Observer, New York, Dance Page Inc., May 1962, p. 70.

Tissot, Roland, Peinture et sculpture aux États-Unis, Paris, Armand Colin, 1973.

Tissot, Roland, L'Amérique et ses peintres, Lyon, Presses universitaires de Lyon, 1990.

Tomkins, Calvin, The Bride and the Bachelors, New York, Penguin, 1976.

\section{NOTES}

1. Nous avons choisi les années 1930 pour parler de modernité dans la danse étatsunienne car cela renvoie à un courant bien spécifique de la danse moderne américaine: le courant correspondant aux critères théoriques avancés par John Martin et représenté dans les années 1930 par Graham et Humphrey principalement.

2. Nous utilisons l'adjectif «denishawnien" pour renvoyer au style de spectacles exotiques et grandioses montés par les deux artistes californiens Ted Shawn et Ruth Saint Denis à partir de 1915. Le circuit des théâtres de vaudeville fournit à la compagnie la majeure partie de ses engagements avec un répertoire de danses hindoues, Indian Suite, de spectacles historiques pompeux interprétés en costumes historiques, Dance Pageant of Egypt, Greece and India.

3. Martha Graham, "Seeking an American Art of the Dance ", in Oliver Sayler, Revolt in the Arts: A Survey of the Creation, Distribution and Appreciation of Art in America, New York, Brentano's Press, 1930, p.250.

4. Tissot, Roland, L'Amérique et ses peintres, Lyon, Presses universitaires de Lyon, 1990.

5. Jean-Michel Rabaté, "La Tradition du neuf, introduction au modernisme anglo-saxon", Cahiers du CNAM, p.106.

6. La citation est traduite par l'auteur de l'article.

7. Martha Graham, Mémoire de la danse, ouvrage traduit de l'américain par Christine Le Bœuf.

8. Premier livre théorique sur le sujet, paru en 1933, regroupant un cycle de conférences données à la New School of Social Research à New York entre 1931 et 1932.

9. Denishawn, école d'arts créée en 1915 à Los Angeles par les chorégraphes pré-modernes Ruth Saint Denis(1877-1968) et Ted Shawn(1891-1972).On étudie, dans cette école, diverses formes de 
danses ethniques, orientales, on cultive l'exotisme et on mène une recherche sur le parallélisme entre danse et musique dans une atmosphère qui mélange mysticisme et show-business. L'enseignement s'étend à tous les arts.

10. Karl Gustav Jung fait une première visite aux États-Unis en 1909, puis y réside régulièrement. Ses écrits sont traduits en anglais dès 1916. De nombreux analystes jungiens exercent sur le sol nord-américain. Graham découvre les écrits de Karl G. Jung au début des années 1930.

11. Citation extraite d'un article intitulé «Votre comportement indien et négroïde : les éléments primitifs de l'esprit américain » paru en 1930 dans la revue Forum.

12. En 1913, à New York, se tient une exposition d'art moderne, l'Armory Show, révélant au public de l'époque le primitivisme des œuvres de Gauguin et ses références polynésiennes, et celui des peintres cubistes tels Picasso et Braque, tous deux influencés par l'art africain.

13. L'idée du primitif englobe chez ces artistes indistinctement Amérindiens, Océaniens, Africains et Afro-américains ; elle possède une dimension plus temporelle que géographique.

14. Louis Horst, pianiste, directeur musical de la Denishawn de 1915 à 1925, part étudier la musique moderne à Vienne où Arnold Schoenberg, Alban Berg et Anton Von Webern bouleversent la tradition musicale en utilisant la théorie de l'atonalité. Il s'intéresse aux philosophes Freud, Arthur Schopenhauer et Friedrich Nietzsche, ainsi qu'aux peintres du Bauhaus. Il transmet ses connaissances de l'art moderne européen aux danseurs étatsuniens Graham, Humphrey et Weidman.

15. Martha Graham, citée par Shea Murphy, The People Have Never Stopped Dancing: Native American Modern Dance History, Minneapolis, University of Minnesota Press, 2007, p.163, citation traduite par l'auteur de l'article.

16. Doris Humphrey, citée par Lloyd, p.86, traduction de l'auteur de l'article.

17. Cette passion pour la nouveauté date de la colonisation lorsqu'Hector St John de Crèvecœur (1735-1813) déclarait, dans Letters from an American Farmer (1782), que tout a eu tendance à régénérer le pionnier : de nouvelles lois, une nouvelle façon de vivre, un nouveau système social. Il est devenu un homme nouveau. St John de Crèvecœur, « What is an American? », Letters from an American Farmer, New York, Dutton, 1877, pp.39-68.

18. John Martin, La Danse moderne, trad. Sonia Schoonejans et Jacqueline Robinson, Arles, Actes Sud, 1991.The Modern Dance, New York, A.S.Barnes and Co., Inc., 1933.

19. Isadora Duncan, citée par Philippe Soupault, Terpsichore, Paris, Hazan, 1928, 1986.

20. Jean-Georges Noverre, Lettres sur la danse et sur les Ballets, Paris, Hachette, 1760, 2012, p.85.

21. Constantin Stanislavski (1863-1938), représentant du «théâtre des mœurs » russe, est rendu célèbre par ses mises en scène naturalistes des pièces d'Anton Tchekhov et de Maxime Gorki. Il est conscient de la puissance évocatrice du mouvement et demande à une danseuse de venir enseigner et créer au sein du Théâtre d'art de Moscou.

22. La traduction de cette citation a été effectuée par l'auteur de cet article.

\section{RÉSUMÉS}

Cet article se propose d'étudier le lien entre la notion de modernité et la danse étatsunienne dans les années 1930. Il s'agira d'entreprendre une analyse des écrits théoriques des chorégraphes Martha Graham (1894-1991) et Doris Humphrey (1895-1958), de commenter certains passages chorégraphiques pour en dégager des signes de modernité. Cette notion est fortement connotée 
et comporte une charge idéologique différente en Europe et aux États-Unis, elle se décline de plusieurs façons : on la trouve dans le contenu et dans la forme, elle peut renvoyer à la quête d'un retour aux sources ou à la recherche de la nouveauté. Elle est un regard porté sur l'histoire, sur le siècle et ses événements.

This article explores the link between the notion of modernity and North-American dance in the 1930's. By studying the various aspects of this concept through an analysis of selected choreographies by Martha Graham (1894-1991)and Doris Humphrey (1895-1958), the pioneers of modern dance, this paper aims to show how modernity, central to these choreographers' aesthetics, pervades their work. The notion of modernity in the United States is both complex and highly connoted, thus difficult to define clearly. Its implications are different in Europe and in the United-States. It refers to a return to the past in order to discover a new form of artistic activity. It also reflects contemporary preoccupations.

Este artículo se propone estudiar la relación entre la noción de modernidad y la danza estadounidense entre 1930 y 1940.Tiene como objetivo realizar un análisis de las obras coreográficas de la época e identificar signos de modernidad. Esta noción tiene connotaciones fuertes y conlleva una carga ideológica diferente en Europa y Estados Unidos, donde ofrece varias caras : se encuentra en el contenido y la forma, y puede refletar la búsqueda de un regreso a los origenes o de la innovación. Es una mirada echada a la historia, al siglo y sus acontecimientos.

\section{INDEX}

Mots-clés : Danse moderne, archaïsme, exotisme, nouveauté, originalité

Palabras claves : Danza moderna, arcaísmo, exotismo, innovación, originalidad

Keywords : Modern dance, archaism, exoticism, novelty, originality

\section{AUTEUR}

\section{CLAUDIE SERVIAN}

Claudie Servian est maître de conférences en civilisation nord-américaine, histoire des idées, histoire de l'art, DETU (directrice des études LEA à Valence) et membre du CEMRA, Université Grenoble-Alpes, Pôle Marguerite Soubeyran, 87, avenue de Romans, 26000 VALENCE, claudie.servian@univ-grenoble-alpes.fr Publications récentes : «Jérôme Robbins, un chorégraphe américain ", Jérôme Benjamin Millepied/Jérôme Robbins, Paris, Opéra de Paris, 2016. "Déconstruction de la tradition », Merce Cunningham/William Forsythe, Paris, Opéra de Paris, 2017 